\title{
Human Robot Team Design
}

\author{
Connor Esterwood \\ School of Information \\ University of Michigan \\ Ann Arbor, Michigan, USA
}

\author{
Lionel P. Robert \\ School of Information \\ Robotics Institute \\ University of Michigan \\ Ann Arbor, Michigan, USA
}

\begin{abstract}
Human-robot teams offer both benefits and new challenges. Human robot teams combine the advantages of automation such as high accuracy, speed, and repeat-ability with the flexibility, adaptability, and creative problem-solving commonly associated with humans. Several challenges, however, must first be addressed to effectively leverage such teams. One challenge is understanding effective human-robot team design (HRTD). HRTD is vital as the wrong team can lead to potentially negative outcomes. The theoretical model and methodology presented are the planned first steps towards the establishment of guidelines based on statistical models that can recommend an optimal human-robot team design based on a given set of criteria.
\end{abstract}

\section{CCS CONCEPTS}

\section{- Human-centered computing $\rightarrow \mathrm{HCI}$ theory, concepts and models.}

\section{KEYWORDS}

HRI; Systematic Review; Personality; Healthcare; Robots; Teams; Teaming

ACM Reference Format:

Connor Esterwood and Lionel P. Robert. 2020. Human Robot Team Design. In Proceedings of the 8th International Conference on Human-Agent Interaction (HAI '20), November 10-13, 2020, Virtual Event, NSW, Australia. ACM, New York, NY, USA, 3 pages. https://doi.org/10.1145/3406499.3418751

\section{BACKGROUND AND INTRODUCTION}

Human robot teams have great potential and offer many different benefits. A human-robot team combines the advantages of automation such as high accuracy, speed, and repeatability with flexibility, adaptability, and the creative problem-solving ability typically associated with humans $[17,19,23]$. Human robot teams are by their very nature "heterogeneous" and if implemented well have the potential to help organizations become more effective and efficient. Human robot teams also have the ability to allow teams to tackle new tasks that were previously unavailable given the physical or cognitive constraints placed on all human teams.

Several challenges, however, must first be addressed to effectively leverage human-robot teams. Teams and teammates are more

Permission to make digital or hard copies of part or all of this work for personal o classroom use is granted without fee provided that copies are not made or distributed for profit or commercial advantage and that copies bear this notice and the full citation on the first page. Copyrights for third-party components of this work must be honored. For all other uses, contact the owner/author(s).

HAI '20, November 10-13, 2020, Virtual Event, NSW, Australia

(c) 2020 Copyright held by the owner/author(s).

ACM ISBN 978-1-4503-8054-6/20/11.

https://doi.org/10.1145/3406499.3418751 than simple work arrangements [15]. Team members provide social support; people generally feel better when they communicate with others who share the same experiences[7, 18]. Humans also enjoy the sense of belonging that comes from participating in a team, especially a team that functions well $[8,16,17]$. This is often reflected in the concept referred to as team cohesion. Cohesion is "a dynamic process reflected in the tendency for a group to stick together and remain united in the pursuit of instrumental objectives and/or the satisfaction of member's affective needs" [1, p.213]. Yet, it is unclear how replacing human teammates with robots might undermine such social support or how best to promote cohesion in these human-robot teams.

Team composition and team size have both been identified as important drivers of successful teams [11, 21]. Human-robot team composition (HRTC) is the ratio of humans to robots in a team; while human-robot team size (HRTS) is the number of agents (i.e. humans and robots) in the team. Research on human teamwork has determined that both of these features are vital to determining the optimal design of teams $[13,17]$. In addition studies have indicated that this may hold true in HRTs as well [4, 5]. Human-Robot teams that are designed incorrectly with the wrong composition and/or size risk undermining: team cohesion, safety, member well-being and team performance $[23,24]$. Therefore, it is vital to design human robot teams with the optimal composition and team size.

The Input-Process-Output (IPO) model is one way to conceptualize the impacts of these variables. In this model, inputs are factors that can be modified or manipulated by designers, engineers, managers, etc $[2,3,20,24]$. Process takes the form of cognitive, emotional, and behavioral actions as well as emergent states that organize independent actions and interactions [3, 9, 12]. Finally, outputs are factors such as performance, safety, efficiency, team member satisfaction and the viability of the team to continue working together [3, 6]. For this study, we utilize a modified version of this model based on previous work in human-robot teamwork conducted by [24]. This proposed model considers inputs as humanrobot team composition and size, process as team cohesion, and output as operational safety, performance, and human well-being. In addition, moderators allow for external factors to be taken into account such as: task types, work settings and other context oriented variables.

Figure 1 presents the paper's research model and depicts the various relationships among variables. The research model can be used to understand how the design of human robot teams can impact teamwork in such teams. In the worst case scenario, poorly designed human robot teams will fail to accomplish their objectives. In the best case scenario, poorly designed human robot teams might lead to minor annoyance. The impact of failure is likely to vary by the team's objective and work setting. For example, in work 


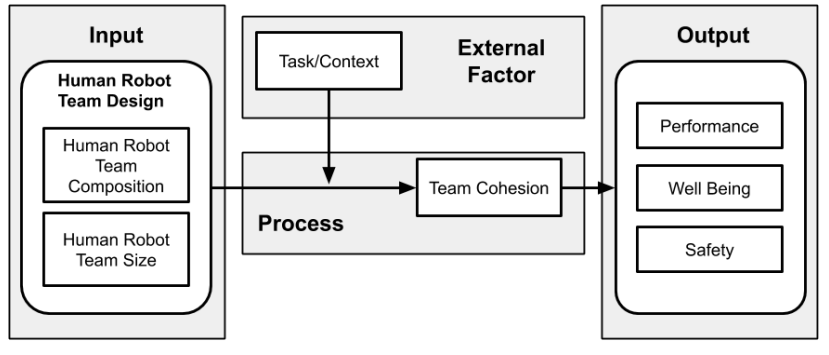

Figure 1: Proposed framework

settings such as long-duration space travel, military applications, and/or search and rescue, failure can lead to a loss of life or injury. Regardless of the work setting minimizing the risk of failure and enhancing safety, performance, and well-being are vital.

\section{EXPERIMENTAL HYPOTHESES}

Hypotheses investigated in this study are informed by the HumanRobot IMOI (Inputs-Mediators-Outputs-Inputs) Team Framework. This framework utilizes sub-components of the IMOI framework of Human-Robot Teamwork proposed in [24]. Based on the propositions in this prior work, we propose the following hypotheses.

H1: The impact of human robot team design (HRTD) will influence performance, team viability, and member well being.

We anticipate outcomes varying as prior research has suggested that team design has a direct impact on the interactions in a given system. $[10,22]$. It is therefore logical that this variability leads to differences in outcomes.

H2: Team cohesion will mediate the impact of HRTD on performance, team viability, and member well being.

We expect team cohesion to have a significant relationship across all groups for each outcome measure. Team cohesion here acts as a process within our proposed IPO model. Prior literature has shown that team cohesion can impact various outcomes and thus we expect it to act as a significant moderator between HRTD and our selected outcomes.

\section{METHOD OF APPROACH}

To investigate the aforementioned aspects of HTRDs, we will adopt a between subject design where each participant in our study will be randomly assigned to one of four experimental conditions. The four conditions in this study take the form of different HRTDs. Specifically we will utilize a $2 \times 2$ design with the following conditions: 2 robots $\& 2$ humans, 1 robot $\& 1$ human, 1 robot $\& 2$ humans, and 2 robots $\& 1$ human. In varying the team size and ratio we are manipulating the overall HRTD to create four unique teams.

\subsection{Task}

To investigate the effects of different HRTDs, we will simulate a collaborative task that will require participants to work as a member of a human-robot team. The goal of each team will be to move certain boxes to an off-screen location while avoiding moving other (I.E. not approved) boxes to that location. The roles within the teams will be fixed for each condition. Humans approve or reject the selected boxes and robot(s) act as the pickers for each box presenting it to the human at specific intervals. The human-robot team will need to move a set number boxes to the off-screen location. This task will remain consistent for each HRTD and only the number of robots or human appearing team members will change.

The pickers and simulated humans will be given a reliable picking rate of over $67 \%$ in accordance with [14]. The exact failure rate will be determined in pilot testing and will remain consistent throughout all conditions. Participants will receive compensation relative to their overall team score. Payment will range by the number of points users gain during the study. The exact range of points to payment will be assessed in a pilot study and after the task and environment's implementation in UnReal. The inclusion of a sliding payment scale acts as an incentive for participants to be engaged in the task and to inject a form of tangible consequence into this study.

\subsection{Apparatus}

This study will be conducted in virtual reality (VR). The VR platform will provide a high degree of immersion in an attempt to best represent a real-world environment. This platform will be developed in the UnReal engine (v.4.25) and is viewed via the Oculus Rift and HTC Vive. Participants in VR will be seated at a table and are given the option to hit one of two buttons on a console. A green button approves the robot's pick in box and a red button rejects it. The virtual environment is a simulated factory environment with ambient noise appropriate to that environment. The UnReal engine was used for its capacity for photo-realism and high fidelity.

For different HRTDs, the number of simultaneous actors within the virtual environment will be varied. For multiple robots, these robots will que behind one another waiting for the human's approval. For multiple humans, the robots will seek approval from both humans. Should the humans disagree, the robot will decide to side with whichever human is correct and if this disagreement should happen multiple times, the robot will alternate between the correct human and the incorrect human. Furthermore, in cases with multiple humans, interactions will be implemented in realtime with humans seeing each other's avatars behind the same table.

\section{DISCUSSION}

The above proposal presents a research project that seeks to inform how humans and robots team together to accomplish different tasks. The concept of human-robot team composition is presented as well as a theoretical model of factors influencing the effectiveness of these teams.Future studies may be conducted that investigate the role of task/context plays in determining the cohesiveness of a given team. The study described in this proposal acts as a first step to this end with the goal of providing a baseline of comparison.

If humans are to indeed start relying on intelligent and versatile robots to extend their own reach and abilities, then there is a need to first determine the optimal design of these teams. The research proposed in this document seeks to provide a platform in the form of model-based guidelines for the design of these teams. 


\section{REFERENCES}

[1] Albert V Carron, Lawrence R Brawley, and William N Widmeyer. 1998. The measurement of cohesiveness in sport groups. Advances in sport and exercise psychology measurement 23, 7 (1998), 213-226.

[2] Susan G Cohen and Diane E Bailey. 1997. What makes teams work: Group effectiveness research from the shop floor to the executive suite. Fournal of management 23, 3 (1997), 239-290.

[3] Leslie A DeChurch, Dorothy R Carter, Raquel Asencio, Amy Wax, Peter W Seely, Kathryn Dalrymple, Sidni A Vaughn, Benjamin R Jones, Gabe Plummer, and Jessica Mesmer-Magnus. 2018. From teams in organizations to organizing in teams. (2018).

[4] Marlena R Fraune, Satoru Kawakami, Selma Sabanovic, P Ravindra S De Silva, and Michio Okada. 2015. Three's company, or a crowd?: The effects of robot number and behavior on HRI in Japan and the USA.. In Robotics: Science and systems.

[5] Marlena R. Fraune, Steven Sherrin, Selma Sabanović, and Eliot R. Smith. 2015 Rabble of Robots Effects: Number and Type of Robots Modulates Attitudes, Emotions, and Stereotypes. In Proceedings of the Tenth Annual ACM/IEEE International Conference on Human-Robot Interaction (Portland, Oregon, USA) (HRI '15). Association for Computing Machinery, 109-116.

[6] JR Hackman. 1983. A normative model of work team effectiveness (No. TR-2). Arlington, VA: Office of Naval Research (1983).

[7] Guido Hertel and Joachim Hüffmeier. 2011. Many cheers make light the work: How social support triggers process gains in teams. Journal of Managerial Psychology (2011)

[8] Harry H Kwon, Galen Trail, and Jeffrey D James. 2007. The mediating role of perceived value: Team identification and purchase intention of team-licensed apparel. Fournal of Sport Management 21, 4 (2007), 540-554.

[9] Jeffery A LePine, Ronald F Piccolo, Christine L Jackson, John E Mathieu, and Jessica R Saul. 2008. A meta-analysis of teamwork processes: tests of a multidimensional model and relationships with team effectiveness criteria. Personnel Psychology 61, 2 (2008), 273-307.

[10] Lanssie Mingyue Ma, Terrence Fong, Mark J Micire, Yun Kyung Kim, and Karen Feigh. 2018. Human-robot teaming: Concepts and components for design. In Field and service robotics. Springer, 649-663.

[11] Dina M. Mansour-Cole. 2007. Cohesion. International encyclopedia of organization studies 1 (2007), 192-194.

[12] Michelle A Marks, John E Mathieu, and Stephen J Zaccaro. 2001. A temporally based framework and taxonomy of team processes. Academy of management review 26, 3 (2001), 356-376.

[13] Michael Muller, Susan R Fussell, Ge Gao, Pamela J Hinds, Nigini Oliveira, Katharina Reinecke, Lionel Robert Jr, Kanya Siangliulue, Volker Wulf, and Chien-Wen Yuan. 2019. Learning from Team and Group Diversity: Nurturing and Benefiting from our Heterogeneity. In Conference Companion Publication of the 2019 on Computer Supported Cooperative Work and Social Computing. 498-505.

[14] Jonathan R Rein, Anthony J Masalonis, Jay Messina, and Ben Willems. 2013. Meta-analysis of the effect of imperfect alert automation on system performance. In Proceedings of the Human Factors and Ergonomics Society Annual Meeting, Vol. 57. SAGE Publications Sage CA: Los Angeles, CA, 280-284.

[15] Lionel Robert and Sangseok You. 2013. Are you satisfied yet? Shared leadership, trust and individual satisfaction in virtual teams. In iConference 2013 Proceedings. 461-466.

[16] Lionel P Robert Jr, Alan R Dennis, and Manju K Ahuja. 2008. Social capital and knowledge integration in digitally enabled teams. Information systems research 19, 3 (2008), 314-334.

[17] Lionel P Robert Jr, Alan R Dennis, and Manju K Ahuja. 2018. Differences are different: Examining the effects of communication media on the impacts of racial and gender diversity in decision-making teams. Information Systems Research 29, 3 (2018), 525-545.

[18] Lawrence B Rosenfeld and Jack M Richman. 1997. Developing effective social support: Team building and the social support process. Fournal of Applied Sport Psychology 9, 1 (1997), 133-153.

[19] Allison Sauppé and Bilge Mutlu. 2014. How social cues shape task coordination and communication. In Proceedings of the 17th ACM conference on Computer supported cooperative work \& social computing. 97-108.

[20] Greg L Stewart. 2006. A meta-analytic review of relationships between team design features and team performance. Journal of management 32, 1 (2006), 29-55.

[21] Michael West and Andreas Richter. 2007. Team Performance. International encyclopedia of organization studies 1 (2007), 1523-1527.

[22] Holly A Yanco and Jill Drury. 2004. Classifying human-robot interaction: an updated taxonomy. In 2004 IEEE International Conference on Systems, Man and Cybernetics (IEEE Cat. No. 04CH37583), Vol. 3. IEEE, 2841-2846.

[23] Sangseok You, Jeong-Hwan Kim, SangHyun Lee, Vineet Kamat, and Lionel P Robert Jr. 2018. Enhancing perceived safety in human-robot collaborative construction using immersive virtual environments. Automation in Construction 96 (2018), 161-170.
[24] Sangseok You and Lionel Robert. 2018. Teaming up with robots: An IMOI (inputsmediators-outputs-inputs) framework of human-robot teamwork. International Journal of Robotic Engineering,(IFRE) 2, 3 (2018). 